\title{
Advanced Video Guidance Sensor (AVGS) development testing
}

\author{
Richard T. Howard ${ }^{a^{*}}$, Albert S. (Nick) Johnston ${ }^{\mathrm{a}}$, Thomas C. Bryan ${ }^{\mathrm{a}}$, and Michael L. Book ${ }^{\mathrm{a}}$ \\ aD19 Simulation Group, NASA - Marshall Space Flight Center, Huntsville, AL, USA 35812
}

\begin{abstract}
NASA's Marshall Space Flight Center was the driving force behind the development of the Advanced Video Guidance Sensor, an active sensor system that provides near-range sensor data as part of an automatic rendezvous and docking system. The sensor determines the relative positions and attitudes between the active sensor and the passive target at ranges up to 300 meters. The AVGS uses laser diodes to illuminate retro-reflectors in the target, a solid-state camera to detect the return from the target, and image capture electronics and a digital signal processor to convert the video information into the relative positions and attitudes. The AVGS will fly as part of the Demonstration of Autonomous Rendezvous Technologies (DART) in October, 2004. This development effort has required a great deal of testing of various sorts at every phase of development. Some of the test efforts included optical characterization of performance with the intended target, thermal vacuum testing, performance tests in long range vacuum facilities, EMI/EMC tests, and performance testing in dynamic situations. The sensor has been shown to track a target at ranges of up to 300 meters, both in vacuum and ambient conditions, to survive and operate during the thermal vacuum cycling specific to the DART mission, to handle EMI well, and to perform well in dynamic situations.
\end{abstract}

Keywords: Automated Rendezvous and Capture, video guidance sensor, AR\&C, AR\&D, VGS, AVGS

\section{INTRODUCTION}

Once the basic design of the Advanced Video Guidance Sensor had been developed, it had to be tested. Initial tests were simply for the purpose of determining how well the basic sensor performed relative to its predicted performance. Later tests were more sophisticated and were aimed at answering more difficult questions. The testing performed with the AVGS during its various stages of development has lead to a greater understanding of its performance and its limitations, and the testing has provided information that was important to refining further tests and improving the sensor.

The AVGS is a sensor designed to acquire and track one or more targets at ranges from $1 / 2$ meter out to 300 meters. The sensor tracks targets that consist of corner-cube retro-reflectors with a filter that passes $850 \mathrm{~nm}$ light and absorbs $800 \mathrm{~nm}$ light. The standard target configuration has a Long Range Target (LRT) and a Short Range Target (SRT), each of which has three retro-reflectors in a line with the center retro-reflector mounted on a pole (to allow small pitch and yaw angles to be measured.) The sensor illuminates the target with $850 \mathrm{~nm}$ light and takes a picture, then illuminates the target with $800 \mathrm{~nm}$ light and takes another picture. The second picture is subtracted from the first picture, and a threshold is subtracted from that value to leave pixels that (mostly) belong to the retro-reflective target (see Figure 1). These target spots are processed, and the information from the spots is used to compute the relative position and range between the target and the sensor. This information is sent out through a serial data port. The operation of the sensor is described in more detail in the papers on the VGS ${ }^{1,2,3}$. The sensor's laser output is in a Gaussian beam that drops to about 1/6 power in the comers of the field-of-view (FOV). And the retro-reflective targets return less light as they are tilted away from the sensor. These optical phenomenon create challenges for the AVGS. The AVGS was designed to track targets at up to a $50 \mathrm{~Hz}$ internal rate, but to save power, there are two tracking rates: Track mode has a $5 \mathrm{~Hz}$ output (with a $10 \mathrm{~Hz}$ internal tracking rate) and Fast Track mode has a $25 \mathrm{~Hz}$ output (with a $50 \mathrm{~Hz}$ internal tracking rate.)

The testing of the AVGS is vital to its successful development. The AVGS is to fly as a part of the Demonstration of Autonomous Rendezvous Technologies (DART) in October, 2004. The AVGS will be the proximity sensor used for the final 300 meters of the approach of the chase vehicle to the target vehicle, a MUBLCOM satellite that was launched in 1999. This target was launched with a modified VGS target mounted on the side, now referred to as the MUBLCOM target (see Figure 2).

"richy.howardianasa.goy; phone 256-544-3536; fax 256-544-8346 
Initial tests were performed using the sensor breadboard. This brassdboard had a pair of laser diodes and a slow data acquisition system connected to a digital camera. The testing was performed at one of the laser test ranges owned by the U.S. Army's Weapons Sciences Directorate, with further testing in the MSFC RF test range. This testing demonstrated that it was indeed feasible to detect spots at ranges of 500 meters or more using this sensor concept, and that at long ranges, the time of the day at which tests occur is important.

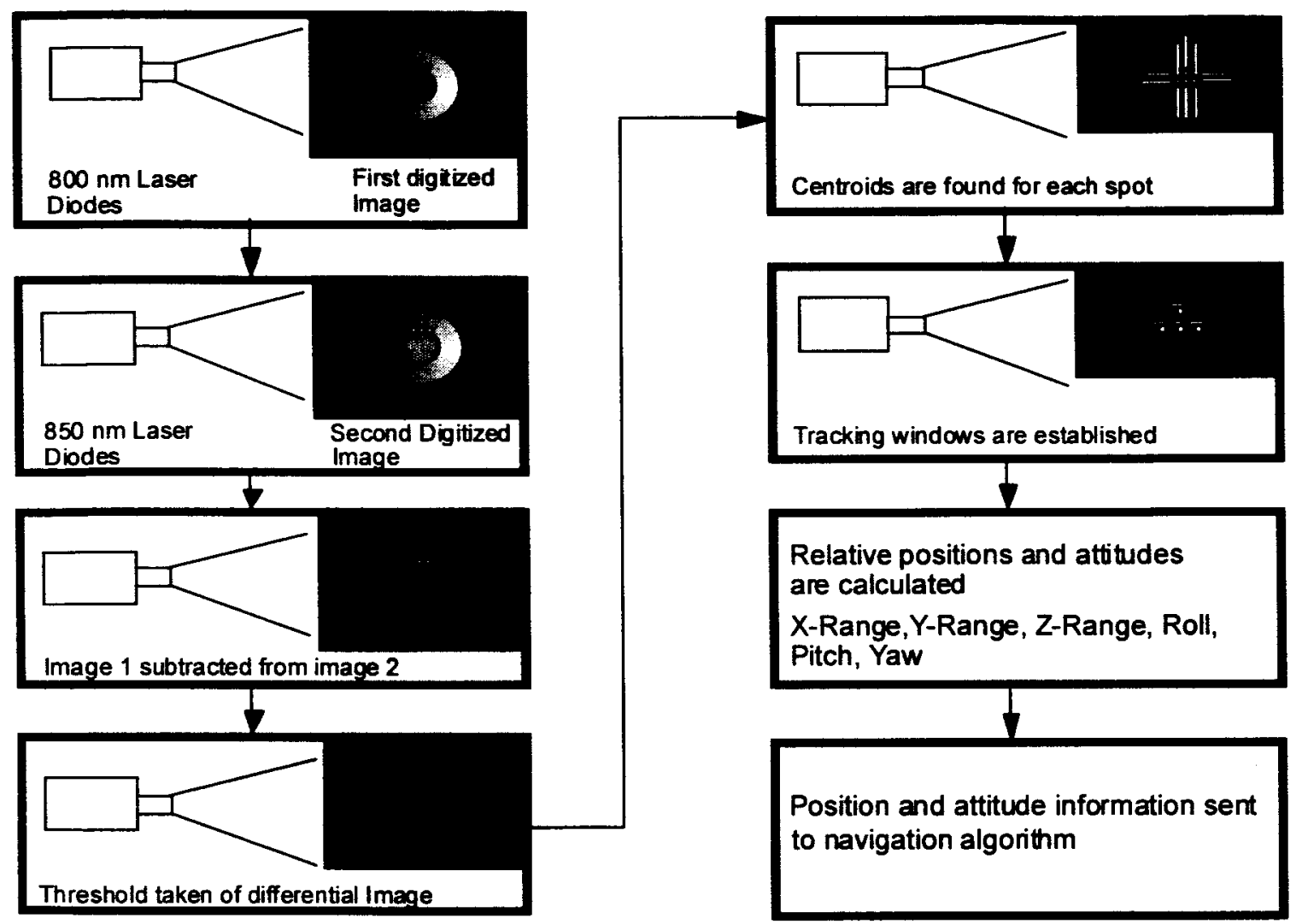

Figure 1: General processing flow of the Advanced Video Guidance Sensor

Once the Initial Prototype (IP) of the AVGS had been completed, much testing was performed to assist in software development and in system performance measurements. The unit could be tested at shorter ranges within the Flight Robotics Laboratory ( $<60$ meters) and in building hallways (up to 200 meters.) One of the important tests that would have to be performed with every AVGS is the Optical Characterization Testing (OCT). It was necessary to know the behavior of the AVGS with respect to different optical parameters. The AVGS has three parameters that it can vary in order to acquire and track a target at different ranges: Integration time (IT) (also known as exposure time or, in a conventional camera, shutter speed), Laser Power (LP), and Threshold (T). It was desired to discover the "best" combination of parameters for each target (a Short Range Target [SRT] usable from ranges of .5 meters to 15 meters or so and a Long Range Target [LRT] usable from ranges of 4 meters out to about 300 meters) at each range.

The Final Prototype (FP) units had several improvements over the IP: there was a metal barrel surrounding the lens opening to restrict EMI and keep the unit cleaner, the firing order of the lasers was reversed, and there were changes to the electronics that collected and processed and compressed the image data before it was transferred to a digital signal processor.

The FP units were completed several months after the IP, and the FP units (FP1 and FP2) were used in much more testing and software development. Some test fixtures were specifically developed to assist with software development (an enclosure that allowed a target to be placed at very short ranges while not exposing any users to laser radiation), 
while other fixtures were used for system testing (a full sized mockup of the target spacecraft and retro-reflectors.) Testing on FP2 included OCT, X-Ray Calibration Facility (XRCF) tests, and many functional tests in the Flight Robotics Laboratory (FRL).

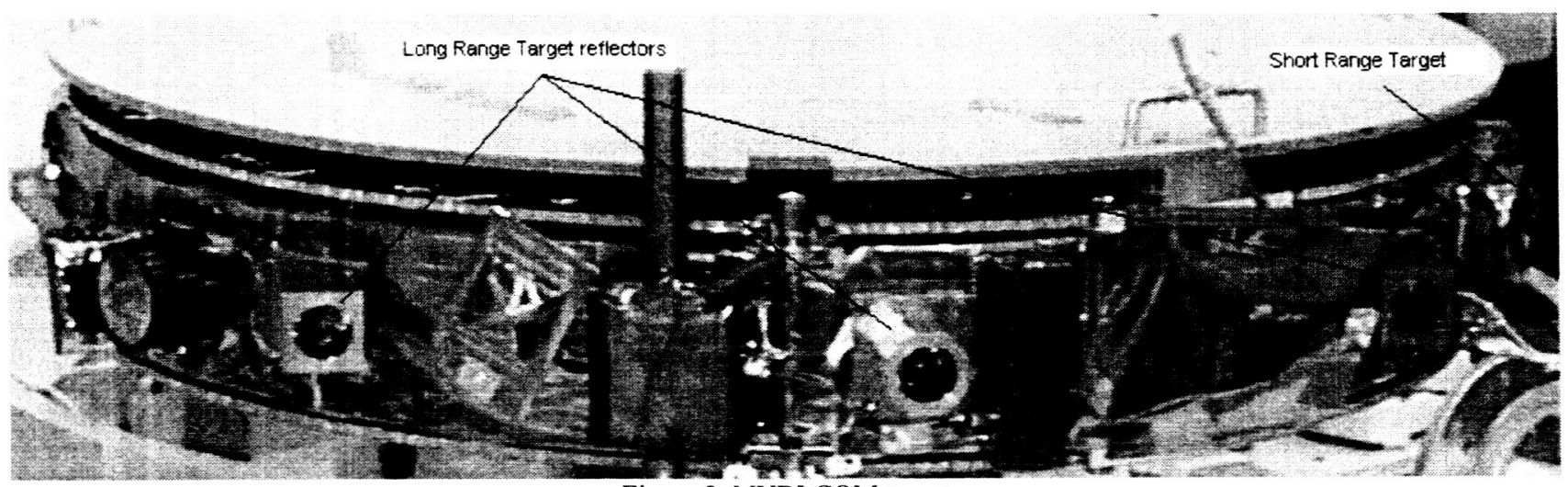

Figure 2: MUBLCOM target

The last units undergoing testing are the flight units. After being completed and functionally tested, the first flight unit was vibration tested and tested in a thermal vacuum environment to ensure that the design was sound and the thermal models were accurate. As of the writing of this paper, Serial Number 2 (SN2) has been delivered to MSFC for testing. The tests performed with this unit to date include OCT, Tracking Performance tests, EMI and EMC tests, tests in the $\mathrm{XRCF}$, tests of the unit's angular tracking rate - the Total Bearing Angular Velocity (TBAV), and dynamic tests in Hardware-in-the-Loop (HWIL) simulations ${ }^{6}$.

\section{DEVELOPMENT TESTS}

During the development of the sensor, the different stages were tested as much as possible. The breadboard system was tested for proof-of-concept, but the first full unit to be tested was the Initial Prototype. The IP testing was followed by development and testing of the Final Prototype units, and that was followed by the development and testing of the flight units.

\subsection{Initial Prototype Testing}

The testing of the IP revolved around determining the best choice of optical parameters for use with the different targets at different ranges. The integration time could be varied to make the targets appear bigger and brighter, the laser power could be varied to make the targets appear bigger and brighter, and the threshold could be varied to decrease the target size and brightness and to eliminate more background noise. As is obvious from the description, all of these parameter's effects were overlapping.

\subsubsection{Spot Mode OCT}

The sensor has six modes of operation, but the two modes used for testing were Spot mode and Tracking mode. In Spot mode, the sensor reports the size, azimuth, and elevation of all of the reflections that it sees in the FOV. In Tracking mode, the sensor tracks the target and reports the relative range, azimuth, elevation, and relative attitude quaternion of the target pattern as well as each of the tracked spots' size, brightness, height, width, azimuth, and elevation.

In Spot mode OCT, the AVGS and target were placed at a known distance away from one another, with the distance being from 2 meters out to 150 meters (the limit of the closest indoor facility). The integration time, threshold, and laser power were varied to create a state space of what combinations created data that would be acceptable for tracking. This state space was a three-dimensional ellipsoid. Unfortunately, when in Spot mode, the spot outputs were sorted by spot size, causing the spots to switch positions in the data stream. This made data analysis difficult. This led to the use of Tracking mode for further OCT testing. 


\subsubsection{Tracking Mode OCT}

In Tracking mode, each tracked reflector was in a dedicated position in the data stream (center spot first, then the top spot, then the bottom spot of the target). This also made the success criteria for a combination of parameters easier to judge. If the AVGS tracked the target 95 percent of the time, it passed. We performed the OCT with the target in the center of the Field-Of-View and with the target at the edge and in the corner. We also performed OCT with the target at different angles from zero degrees yaw and pitch up to 25 degrees yaw and pitch. As can be seen, there are MANY combinations of variable that must be tested in order to completely test the sensor, and time was limited.

\subsection{Final Prototype OCT}

Once the FP units of the AVGS had been delivered to the Marshall Space Flight Center, they were immediately put to use for OCT testing and software development. FP2 was the unit used to determine the optical parameters to be used in the software for tracking the targets. The method for this OCT testing was the same as the final method used with the IP: Tracking Mode OCT. But there was a large test space for this unit, so the testing took several months. During this time, other methods of performing OCT were discussed, but due to other circumstances, the method was not changed. There were some anomalies from this testing, and they are laid out in the data and results section of this paper.

\subsection{SN2 OCT \& Tracking Performance Tests}

Before the first flight unit AVGS arrived at MSFC, a new method of OCT testing was devised. First, based on previous testing, a threshold was picked for use at every range. The fixing of a threshold greatly dropped the test space necessary for OCT. Then, based on the physics of the AVGS (the laser return varies with the inverse square of the range), the laser power output was computed based on range such that the unit puts out minimal laser power (10\%) at close range (3 meters) and maximum laser power at long ranges (greater than 200 meters), with the power at 100 meters being one fourth the maximum power. This fixing of two of the three parameters led to a much smaller test space. The only variable remaining, integration time, was chosen by testing a target in the corner of the FOV at each range with a very high threshold. The target was tilted at the maximum specified value (to minimize the return from the retro-reflectors), and the comer was chosen in order to make sure the system worked in the dimmest portion of the FOV. Then the unit was commanded to view the target with a range of integration times. The minimum integration time that lead to an acceptable target pattern was chosen as the best value for that range.

During this time, it became apparent that the flight units would have to be characterized and tested with ranges beyond the $150 \mathrm{~m}$ available in the FRL building. With the assistance and support of the MSFC facilities personnel and Propulsion Test Area personnel, the cable tunnel to the historic Saturn I test stand was cleared and reactivated to provide a straight underground tunnel for testing at ranges up to $300 \mathrm{~m}$ for sensor characterization and testing.

Unfortunately, at very long ranges ( $>150$ meters), there were problems with this approach. At long ranges, the target spots appear very close together, and when the sensor was tested with a very large threshold and then run using a lower threshold, the spots would merge together, causing the AVGS to not be able to track the target. Also, due to the variability in laser output across the FOV, the integration time that would allow the target to be tracked in the corner of the FOV would not work in the center of the FOV, and vice versa. So for longer ranges, an average of the maximum and minimum acceptable integration times in the center of the FOV was chosen as the value to use at the range under test. This gave the most robust parameter set.

Once the OCT had been finished, the flight software was modified to utilize the parameters that had been determined from the OCT testing. Then, the Tracking Performance Tests began. The Tracking Performance Tests were performed in order to quantify several measures of the sensor's performance, such as the noise levels of the sensor at different ranges, angles, and target attitudes. These tests consisted of running the sensor in Track mode at approximately 25 different combinations of target azimuth, elevation, and attitude as well as running some special tests to determine the bounds of the sensor's performance. The special tests were different types of field-of-view tests in which either the sensor or the target was pitched and yawed to determine the maximum angles at which the AVGS could still track the target at the range under test. The Tracking Performance Tests were run at about 10 different ranges, from 4 meters out to 300 meters. 


\subsection{X-Ray Calibration Facility testing}

There were some fundamental questions on the AVGS project that concerned AVGS design and performance: 1) "Does spot size measured by the AVGS change between ambient and vacuum enough to affect tracking"? From previous flight tests (Shuttle flights STS-87 and STS-95) with the VGS, the measured spot sizes changed due to vacuum effects ${ }^{4,5}$, and the sensor had had difficulties with tracking the SRT at 10 meters due to the spot size change.

2) "Can the sensor detect a target at 500 meters or more?" During the flight experiment, the AVGS will be commanded to acquire the target at up to $1000 \mathrm{~m}$ range.

3) "Can range be determined at 500 meters?" It was desirable for the sensor to be able to detect separate spots at even longer ranges than the nominal sensor specification of 300 meters.

The X-Ray Calibration Facility was uniquely qualified to help answer these questions. The XRCF was designed for Xray calibration and performance testing of grazing angle $\mathrm{x}$-ray optics and detectors and for cryogenic interferometric testing of direct-incidence optics. It features a 518 meter vacuum tube and a large vacuum chamber $(6 \mathrm{~m} . \times 18.2 \mathrm{~m}$.) at the end. A separate building is used as an x-ray source, while other buildings house support equipment. For the AVGS testing, the tube and some of the support buildings were utilized. See Figure 3. Because of equipment conflicts, the furthest distance AVGS could be tested was $483 \mathrm{~m}$.

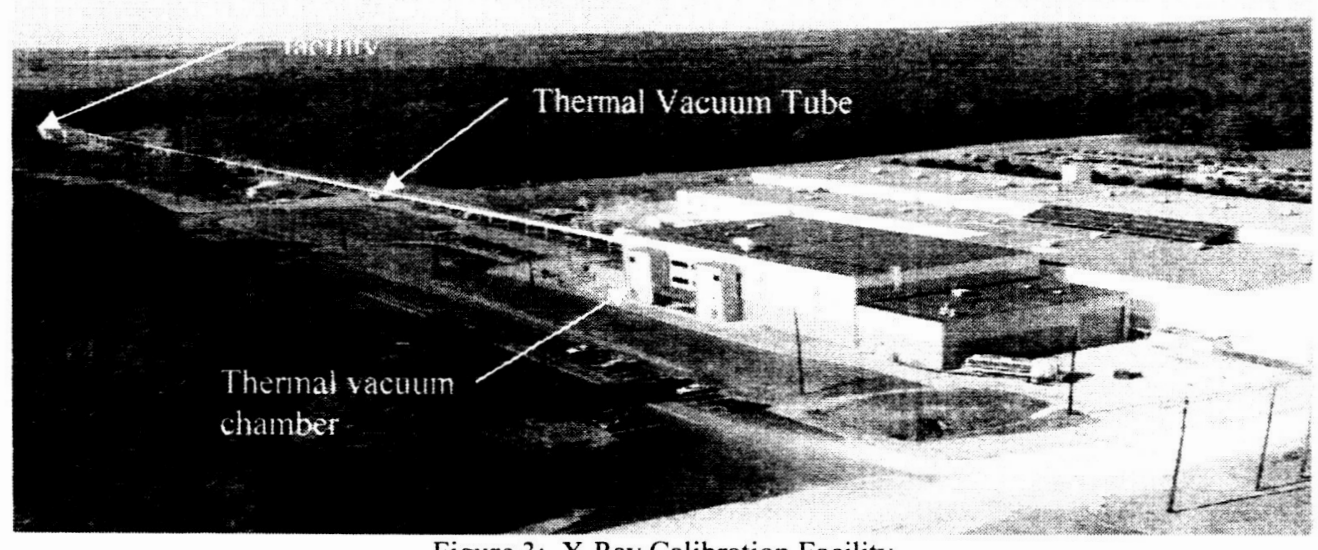

Figure 3: X-Ray Calibration Facility

The sensor was physically located in Bldg. 4718-2. A manway provided access into the XRCF tube and the sensor was mounted on a special test fixture. For the FP2 test, additional thermal sensors were added for thermal insight. On the SN2 these sensors were re-mounted and used again. (See Figure 4)

The target was mounted at different ranges down the tube. XRCF personnel would physically move the target to the proper location and set it up using facility drawings for position. For the FP2, a laser rangefinder was used to doublecheck target placement, but since the XRCF drawings were accurate enough (within 6 inches normally) the laser rangefinder was only used for close ranges for SN2. This target did differ from the AVGS on-orbit target in that the center retro-reflector was not mounted on a pole (see Figure 5).

The target could be mounted directly on the curved interior tube of the XRCF, and a curved top could be placed on the target to have it conform to the top of the tube. The tube widens at different points, and legs can be attached to both the top and the bottom of the target curves in order to keep the target centered in the tube (see Figure 6). 


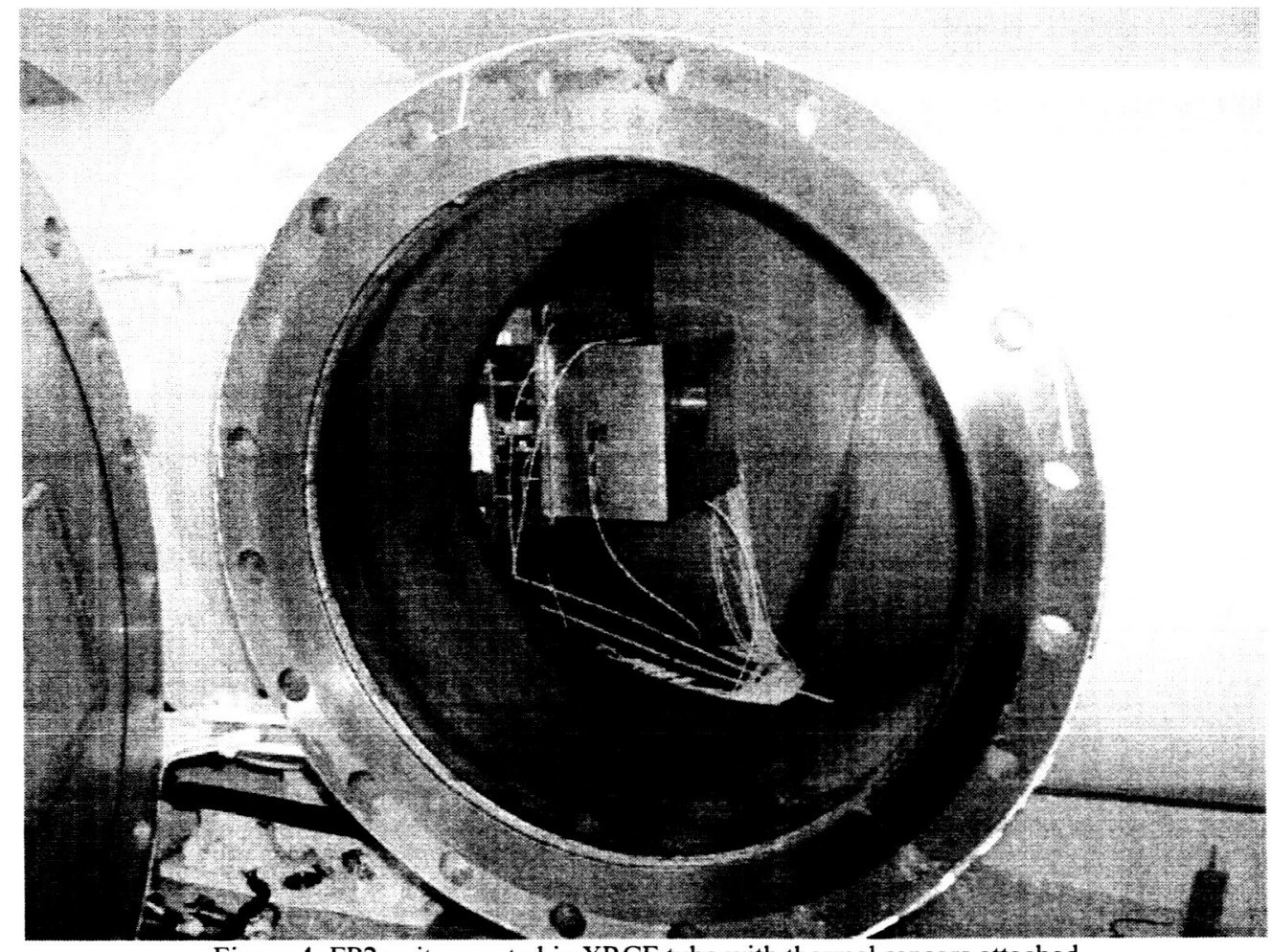

Figure 4: FP2 unit mounted in XRCF tube with thermal sensors attached
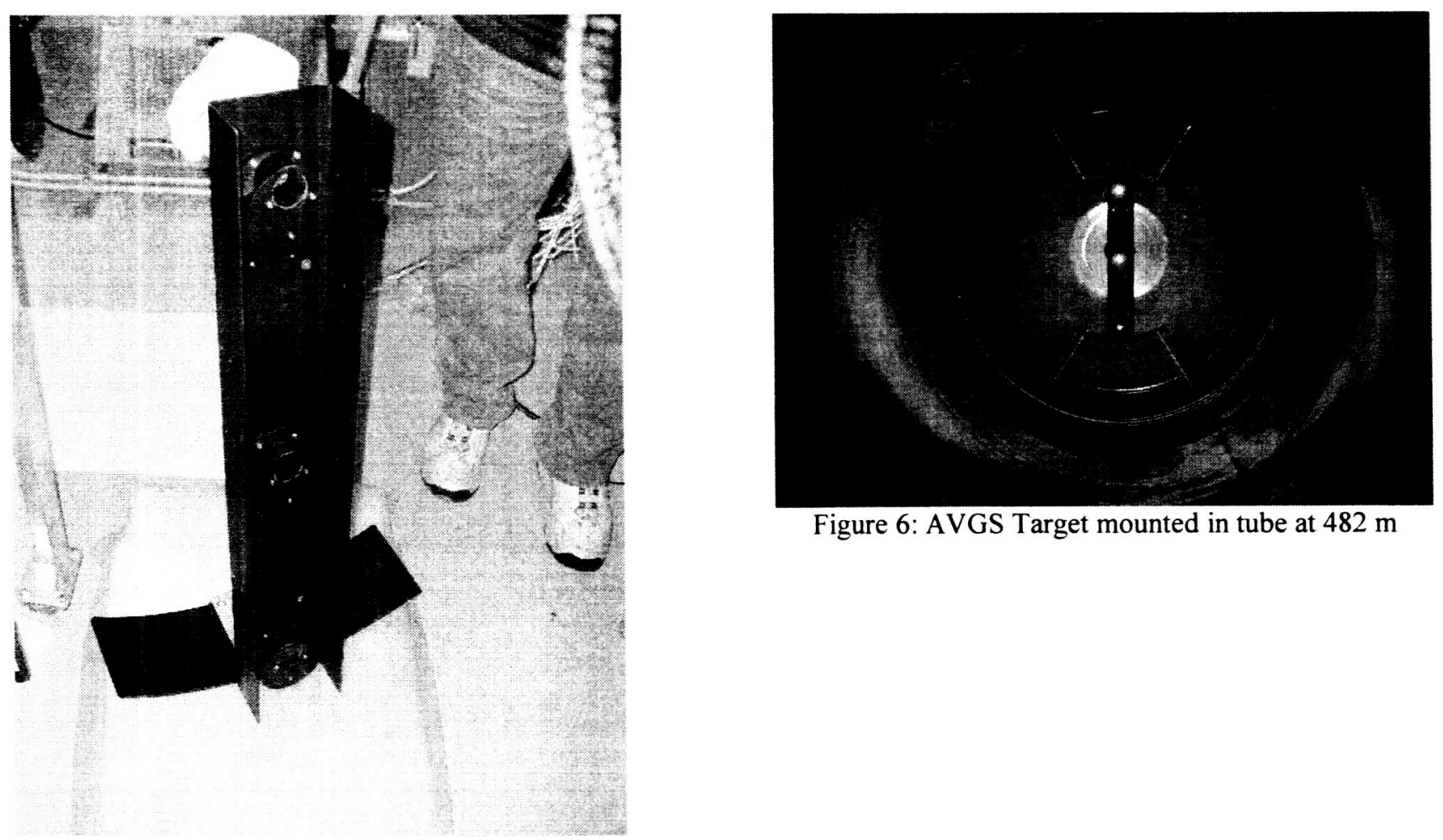

Figure 6: AVGS Target mounted in tube at $482 \mathrm{~m}$

Figure 5: AVGS Target used for XRCF testing (with the top mount removed for clarity) 


\subsection{TBAV Tests}

The AVGS was required to acquire a target moving at $1 \%$ second in azimuth and elevation in the sensor's field-of-view and to track a target moving up to $2 \%$ second in those axes. The test apparatus used to verify this requirement was the Advanced Sensor Optical Calibration Robot, or ASOCR. ASOCR is a 5 DOF robot capable of moving a target to various positions (denoted as a $\mathrm{x}, \mathrm{y}$, roll, pitch, and yaw). The translation rails are Hauser HLE100c's with stepper motors and encoders on every DOF. ASOCR's capabilities are tabulated in Table 1.1.

\section{ASOCR Abilities Table 1.1}

Translation

$13.76 \times 2.54$ meters (railing length)

$11.48 \times 1.67$ meters (current limit switches, adj.)

Max speed $5 \mathrm{~m} / \mathrm{s}$

Max acceleration $10 \mathrm{~m} / \mathrm{s}$

Repeatability $+/-.2 \mathrm{~mm}$

Roll

$+/-12$ (software)

+/- 20 (hardware) (Also target dependent, can be infinite with small target)

Max rotation $15 \mathrm{rev} / \mathrm{s}$

Max angular acceleration $30 \mathrm{rev} / \mathrm{s} / \mathrm{s}$

Repeatability .5 arc min
Pitch

\begin{tabular}{|l|}
\hline$+/-30 \mathrm{deg}$ (limit switches) \\
\hline$+/-90 \mathrm{deg}$ (hardware limited) \\
\hline Max rotation $15 \mathrm{rev} / \mathrm{s}$ \\
\hline Max angular acceleration $30 \mathrm{rev} / \mathrm{s} / \mathrm{s}$ \\
\hline Repeatability .5 arc min \\
\hline
\end{tabular}

\begin{tabular}{|l|}
\multicolumn{1}{c|}{ Yaw } \\
\hline$+/-30$ deg (limit switches) \\
\hline$+/-90 \mathrm{deg}$ (hardware limited) \\
\hline Max rotation $15 \mathrm{rev} / \mathrm{s}$ \\
\hline Max angular acceleration $30 \mathrm{rev} / \mathrm{s} / \mathrm{s}$ \\
\hline Repeatability .5 arc $\mathrm{min}$ \\
\hline
\end{tabular}

For the AVGS testing the sensor was also located on a movable head (azimuth and elevation). The 5 DOF of ASOCR and the pitch capability (elevation) of the test stand was computer controlled with stepper motors for repeatable movements and controlled speed.

For the TBAV test, the sensor elevation was moved to $-8.5^{\circ}$ and then commanded to move upward at a rate of $2 \%$ second while the target was stationary and the sensor was in Track mode. Then the same test was run with the sensor in Fast Track mode, to make sure the TBAV requirement was met during either type of Track mode.

\subsection{Thermal Vacuum}

The testing of the AVGS in a thermal vacuum chamber was required as part of the acceptance test before the unit could be declared fully functional. The test consisted of eight thermal cycles while in a vacuum, with temperatures ranging between the expected highs and lows of the DART mission. The maximum survival temperature tested was 61 degrees $\mathrm{C}$, and the maximum operational temperature tested was 40 degrees $\mathrm{C}$, and the minimum temperature reached was -24 degrees C. During the test, the AVGS was run in both Standby mode (just to show that it was alive) and in Tracking mode (to show that it was functioning properly.)

\subsection{Electro-Magnetic Interference/Electro-Magnetic Compatibility (EMI/EMC)}

Various operational anomalies were seen during the OCT and Tracking Performance testing of the AVGS SN2. The anomalies involved checksum errors in communications as well as occasional unexpected error bits being set, and even, on rare occasions, an uncommanded system reset. One theory was that external EM fields or signals were actually causing these anomalies, and that the number of anomalies would increase when the AVGS was integrated on the 
spacecraft. Also, since the AVGS EMI Qualification testing was not scheduled until installation of the flight unit, a developmental EMI Test was run.

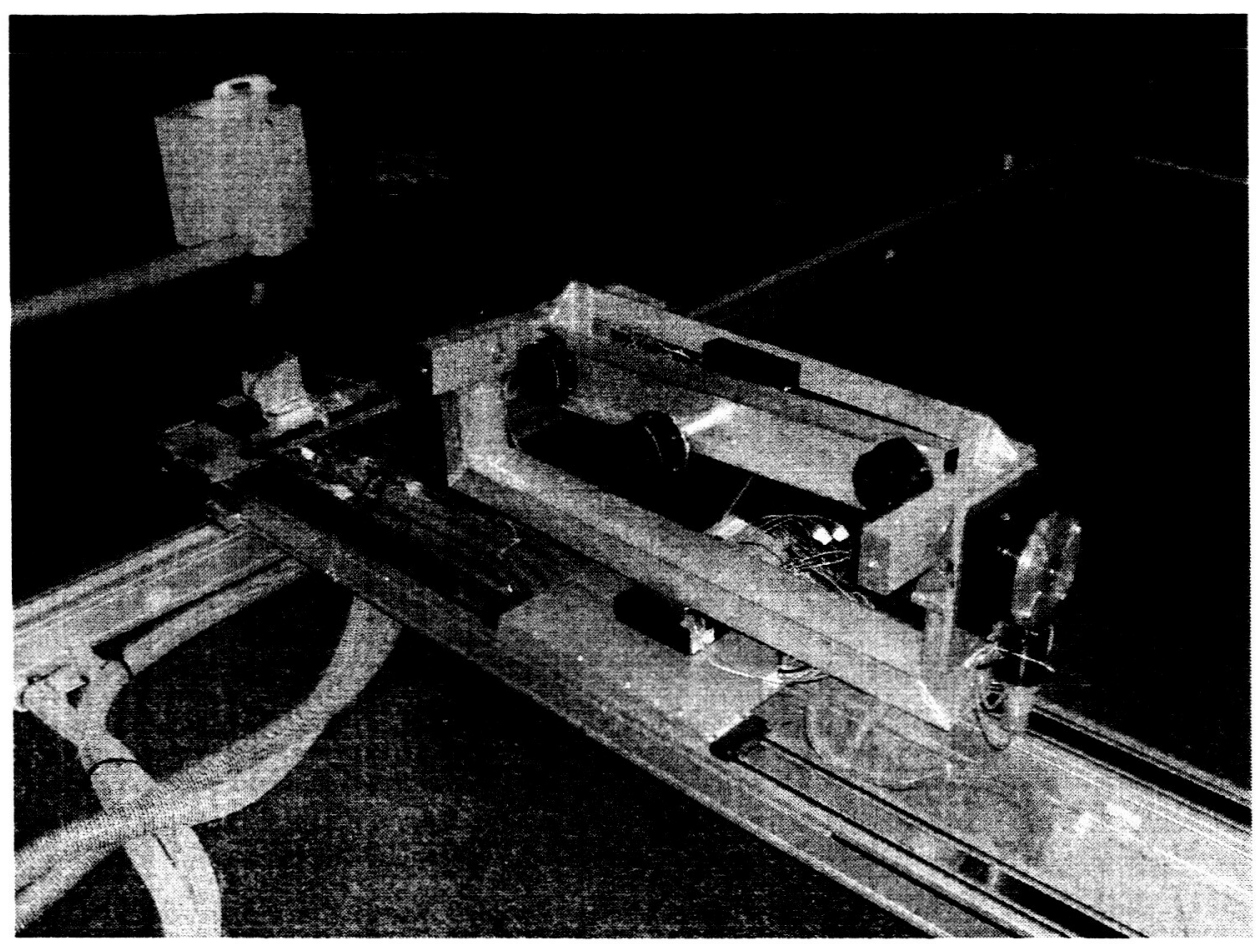

Figure 7: ASOCR with AVGS Long Range Target

\section{DATA AND RESULTS}

\subsection{IP OCT}

The data from the Initial Prototype OCT tests was inconclusive. The parameter space was essentially a very long, narrow cigar shape, so picking the "best" combination was somewhat subjective. And to complicate matters, there was the issue that the results of the tests changed over time. It turned out that the laser diodes were dropping in power. When they were tested, one of the two foreground lasers was at half power, and the other was entirely dead. The dead laser, along with similar problems with FP2, caused an intense investigation of the lasers and failure modes, with the result being a change in the laser driver circuitry in the flight units, and a modification to the FP units.

\subsubsection{IP Spot Mode OCT}

The Spot Mode OCT was simply too difficult to perform effectively.

\subsubsection{IP Tracking Mode OCT}

This method of performing OCT was very time intensive, and it was decided that a better method was needed in the very near future (for the testing of the FP units once they had arrived.) Also, the test results varied slightly from run-to-run. But even more disturbing was that over time, the tests varied even more. So when a test was rerun some weeks after an initial run, the results were different. It turned out that the initial design of the laser drivers did not have sufficient filtering, and the lasers were initially damaged and slowly degrading over time as a result of turn-on transients. 


\subsection{FP OCT}

After much testing, a set of optical parameters was derived for use in the FP2 unit. These parameters worked for every range at which they were tested (which was only up to about 60 meters - the limit of our laboratory.) The same set of parameters was loaded into FP1, and the unit would not acquire or track the target. Upon investigation, it was discovered that FP1 had much brighter lasers than FP2, so the target spots were too large to be recognized by the software as being valid spots for the range in question. A new set of parameters was created for FP1 by scaling the integration times from the FP2 parameters. This set of parameters worked well in the FP1 testing, and they were then used in the first flight unit, SN2. They worked just as well in SN2 as they had in FP1, which was an encouraging result after the problem of FP1 and FP2 not being equal in performance.

\subsection{SN2 OCT \& Tracking Performance Tests}

The results of the SN2 OCT testing were a set of parameters that were to be used by the sensor at every range in order to acquire and track the target. These parameters were programmed into the flight software and tested in the Tracking Performance tests.

The first result of the Tracking Performance tests was that the AVGS did indeed track with the parameters that had been discovered during OCT. The second result of the tests was that the noise levels of the sensor were far lower than had been modeled by the guidance, navigation, and control (GN\&C) teams that were responsible for using the AVGS data as the input to their systems. The data from these tests is still being analyzed.

\subsection{XRCF}

The XRCF testing generated large amounts of data. The FP testing created much information that was used to refine some of the flight unit designs as well as to compare with later flight unit testing.

\subsubsection{FP Results}

The initial FP test ran from August 11, 2003 until September 4 at the following ranges (meters): 1,3.2, 4, 5, 208, and 300. Also different target configurations and sensor alignments were used. Spot variations were so great that the vacuum/ambient changes were lost in the noise. Some of the problems discovered during or after the test were: lens hazing, firmware software algorithms, imager cold finger debonding, laser fiber misalignment, and solar filter band pass errors and optical aberrations induced by the solar filter. Since all the hardware or software problems precluded a good test, the test was stopped to work on the AVGS.

\subsubsection{SN2}

The S/N 002 sensor performed well in testing. The box was tested at the following ranges (meters): 5, 15, 200, 300, and 482. At $482 \mathrm{~m}$, the target with three retroreflectors was successfully detected, which answered question 2 ) at the beginning of this section. The center retroreflector was covered, and the ability to resolve two separate targets meant (with the proper retro spacing) range could be resolved well past 300 meters, answering question 3 ). An early analysis of the spot data still shows enough variation that the ambient/vacuum effects are in the noise level of other variations and should not affect tracking or the mission. 


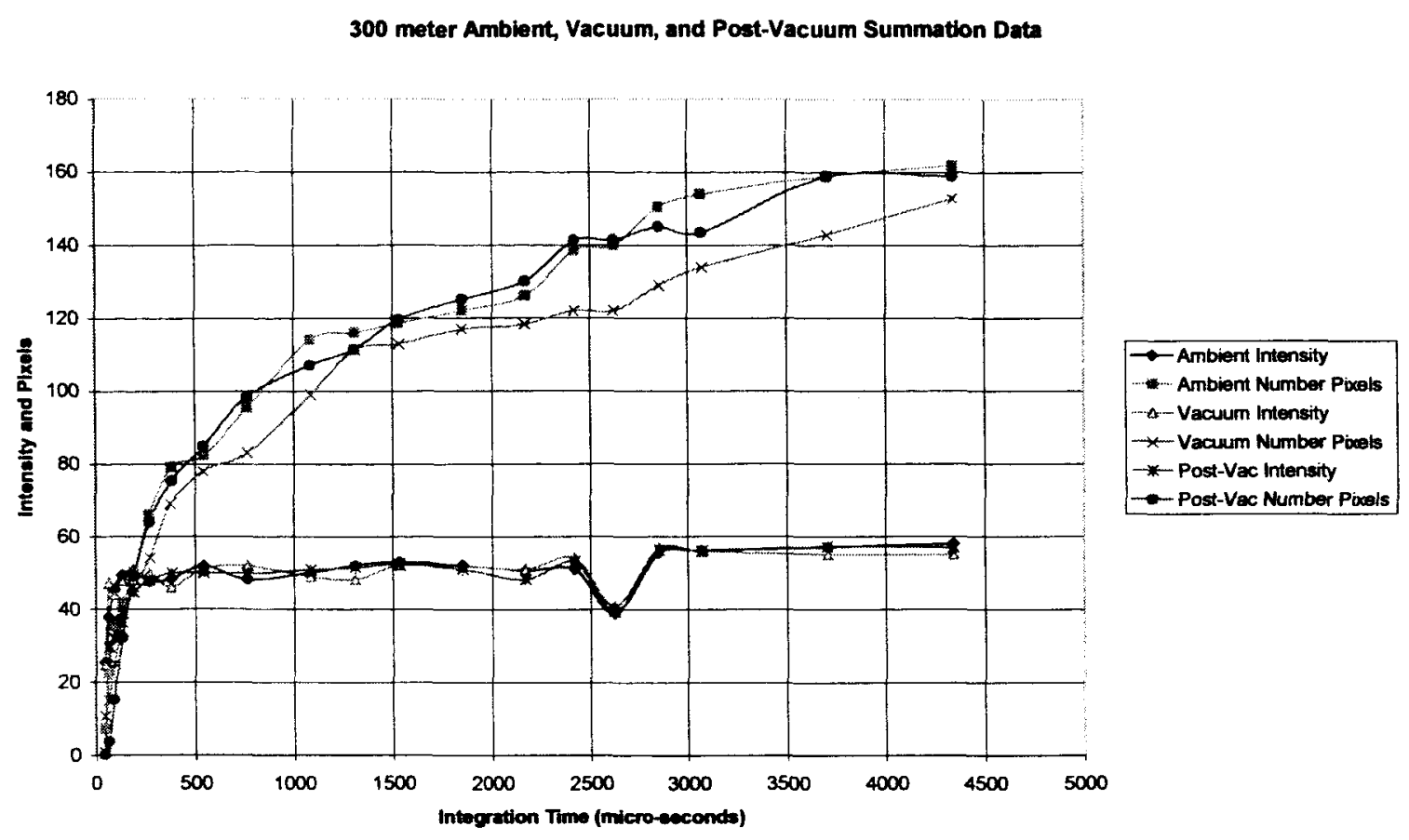

Figure 9: Comparison of spot sizes versus integration time under different conditions in the XRCF

\subsection{TBAV Test Results}

The $5 \mathrm{~Hz}$ data was saved in a file (20040219180813_TBAV_ST.dat) and the fast track data in a separate file (20040219181751_TBAV_FT.dat). Both are located on the AVGS server at MSFC. Both show tracking without loss, and therefore this test was successfully completed.

\subsection{Thermal Vacuum}

The sensor took several tries before finally passing the thermal vacuum test. There were several problems with various thermal components, but each of the reworks was performed quickly. The first problem was a temperature sensor that debonded inside the unit, causing a thermal shutdown as the sensor cooled. Once the sensor bonding process had been corrected, the sensor again went into thermal vacuum testing, and it failed on the hot side two separate time. First, the thermo-electric coolers (TECs) had too much thermally conductive epoxy around them, causing a thermal short, so they were not providing any cooling, and a second time it was discovered that an electrical connection inside the AVGS was too close to a hot part of the box, and the connection was carrying heat back into a component that was supposed to stay cool. Once these issues were cleared up, the unit operated very well at its designed temperature extremes.

\subsection{EMIEMC Results}

The first test run was the Radiated Emissions test. This test allowed some changes to cable shielding and grounding to be made and tested. The results were mixed, with overall emissions exceeding the specified levels, but the AVGS emissions not exceeding the guard limits for the on-board UHF and GPS receivers.

The next test was for radiated susceptibility to external signals, including the on-board S-Band transmitters, which had little or no detectable effect on the AVGS, which did not have its usual GSE boxes attached.

The next test was for Conducted susceptibility to noise on power buss or magnetically coupled in, which also had little or no detectable effect on the AVGS. 
The final test was for Conducted Emissions to detect the noise the AVGS will put back on the power buss. This test showed some leakage of the switching noise that the internal DC/DC power converters generate.

Overall, the AVGS seemed to be relatively immune to outside EMI when configured for flight and not too noisy to its avionics neighbors.

\section{CONCLUSIONS}

Testing is vital to the development and characterization of hardware. The tests performed on the AVGS during its various stages of development helped define its performance and show its limitations as well as uncover unexpected pitfalls. The behaviors uncovered during testing have helped improve the overall sensor performance and robustness. Extensive testing should be performed whenever possible in order to improve the unit under development.

\section{ACKNOWLEDGMENTS}

The authors wish to thank the facilities described in all of the tests above: the X-Ray Calibration Facility, the Flight Robotics Laboratory, the Electro-Magnetic Compatibility Test Lab, the Test Area, the MSFC RF test range, and the Weapons Sciences Directorate. The authors also wish to thank their management for their support and for the opportunity to work on the systems and facilities described in this paper.

\section{ACRONYMS AND ABBREVIATIONS}

\begin{tabular}{llll}
\multicolumn{2}{c}{ Automatic Rendezvous \& } & IP & $\begin{array}{l}\text { Initial Prototype } \\
\text { International Space Station }\end{array}$ \\
AR\&C/D/M Capture/Docking/Mating & ISS & Marshall Space Flight Center \\
AVGS & Advanced Video Guidance Sensor & MSFC & $\begin{array}{l}\text { National Aeronautics and Space } \\
\text { Demonstration Automatic Rendezvous }\end{array}$ \\
DART & $\begin{array}{l}\text { Technology } \\
\text { Degres of Freedom }\end{array}$ & NASA & Administration \\
DOF & OCT & Optical Characterization Testing \\
DOTS & Dynamic Overhead Target Simulator & SN & Serial Number \\
FP & Final Prototype & STS & Space Transportation System \\
FRL & Flight Robotics Laboratory & VGS & Video Guidance Sensor \\
GN\&C & Guidance Navigation and Control & XRCF & X-Ray Calibration Facility \\
GPS & Global Positioning System & & \\
HWIL & Hardware-in-the-Loop & &
\end{tabular}

\section{REFERENCES}

1. R. Howard, H. Cole, J. Jackson, G. Kamerman, D. Fronek, "Automatic Rendezvous and Docking System Test and Evaluation", Proceedings of SPIE Laser Radar Technology and Applications II, pp. 131-139, 1997.

2. R. Howard and M. Book, "Improved Video Guidance Sensor for Automatic Docking", Proceedings of SPIE Space Guidance, Control, and Tracking II, pp. 118-127, 1995.

3. R. Howard, T. Bryan, M. Book, and J. Jackson, "Active Sensor System for Automatic Rendezvous and Docking", Proceedings of SPIE Laser Radar Technology and Applications II, pp. 106-115, 1997.

4. R. Howard, T. Bryan, and M. Book, "Video Guidance Sensor Flight Experiment Results", Proceedings of SPIE Laser Radar Technology and Applications III, Vol. 3380, pp. 315-325, 1998.

5. R. Howard, T. Bryan, M. Book, and R. Dabney, "The Video Guidance Sensor - A Flight Proven Technology", Proceeding of the $22^{\text {nd }}$ Annual American Astronautical Society Guidance and Control Conference, Vol. 101, pp. 281298, 1999.

6. F. Roe, R. Howard, and L. Murphy, "Automated Rendezvous and Capture System Development and Simulation for NASA", Proceedings of SPIE Space Technologies and Operations conference, 2004. 